\title{
Structure and organization of phycobilisomes on membranes of the red alga Porphyridium cruentum
}

\author{
Ana A. Arteni · Lu-Ning Liu · Thijs J. Aartsma · Yu-Zhong Zhang • \\ Bai-Cheng Zhou $\cdot$ Egbert J. Boekema
}

Received: 10 September 2007/ Accepted: 11 September 2007/Published online: 9 October 2007

(C) Springer Science+Business Media B.V. 2007

\begin{abstract}
In the present work, electron microscopy and single particle averaging was performed to investigate the supramolecular architecture of hemiellipsoidal phycobilisomes from the unicellular red alga Porphyridium cruentum. The dimensions were measured as $60 \times 41 \times$ $34 \mathrm{~nm}$ (length $\times$ width $\times$ height) for randomly ordered phycobilisomes, seen under high-light conditions. The hemiellipsoidal phycobilisomes were found to have a relatively flexible conformation. In closely packed semicrystalline arrays, observed under low-light conditions, the width is reduced to 31 or $35 \mathrm{~nm}$, about twice the width of the phycobilisome of the cyanobacterium Synechocystis sp. PCC 6803. Since the latter size matches the width of dimeric PSII, we suggest that one PBS lines up with one PSII dimer in cyanobacteria. In red algae, a similar 1:1 ratio under low-light conditions may indicate that the red algal phycobilisome is enlarged by a membrane-bound peripheral antenna which is absent in cyanobacteria.
\end{abstract}

Ana A. Arteni and Lu-Ning Liu equally contributed to the work.

Electronic supplementary material The online version of this article (doi:10.1007/s11120-007-9264-z) contains supplementary material, which is available to authorized users.

A. A. Arteni - E. J. Boekema

Department of Biophysical Chemistry, GBB, Rijksuniversiteit Groningen, Groningen, The Netherlands

L.-N. Liu · T. J. Aartsma ( $₫)$

Biophysics Department, Huygens Laboratory, Leiden

University, Leiden, The Netherlands

e-mail: aartsma@physics.leidenuniv.nl

L.-N. Liu · Y.-Z. Zhang · B.-C. Zhou

State Key Lab of Microbiol Technology, Shandong University, Jinan 250100, P. R. China
Keywords Phycobilisome - Red alga ·

Porphyridium cruentum - Electron microscopy ·

Single particle analysis

$\begin{array}{ll}\text { Abbreviations } \\ \text { APC } & \text { Allophycocyanin } \\ \text { Chl } & \text { Chlorophyll } \\ \text { EM } & \text { Electron microscopy } \\ \text { FRAP } & \text { Fluorescence recovery after photobleaching } \\ \text { PBS } & \text { Phycobilisome } \\ \text { PC } & \text { Phycocyanins } \\ \text { PE } & \text { Phycoerythrins } \\ \text { PSI } & \text { Photosystem I } \\ \text { PSII } & \text { Photosystem II }\end{array}$

\section{Introduction}

In cyanobacteria and red algae, the main accessory lightharvesting complexes are the phycobilisomes (PBSs), large and highly structured assemblies of phycobiliproteins associated to the cytoplasmic surface of the thylakoid membrane (Gantt 1980; Grossman et al. 1993; MacColl 1998; Adir 2005). They capture and transfer energy primarily to chlorophyll (Chl) $a$ in Photosystem (PS) II (Gantt 1986; Grossman et al. 1993, 1995; Sidler 1994; MacColl 1998; Samsonoff and MacColl 2001; Adir 2005). The PBSs are composed of $\alpha$ - and $\beta$ - polypeptides bearing covalently attached open-chain tetrapyrroles (Betz 1997). They assemble in disc-shaped aggregates that are stacked in the peripheral rods of the PBS. Colourless linker polypeptides between adjacent discs stabilize the PBS structure and regulate energy transfer (Liu et al. 2005). 
PBSs in the unicellular red alga Porphyridium cruentum were first observed about 40 years ago (Gantt and Conti 1966) and described as hemiellipsoidal. Based on electron microscopic results, a preliminary model was proposed (Gantt et al. 1976) involving a tricylindrical core subassembly in the semi-spherical centre and several peripheral rods radiating out from the centre to form the rounded surface of the semi-sphere. The core contains allophycocyanin (APC), while the peripheral rods are mainly composed of phycocyanins (PC) and phycoerythrins (PE). In other systems, hemidiscoidal PBSs have been identified of which the 3-D structure was recently characterized in some detail by single-particle electron microscopy (Barber et al. 2003; Yi et al. 2005). However, such detailed information of hemiellipsoidal PBSs is still lacking.

Despite the fact that energy transfer and mutational studies have indicated that phycobilisomes can couple directly to PSI as well as to PSII, little is known about the structural aspects of the interaction. The precise association of phycobilisomes to PSII and PSI is not known, but there are some hints which PSII subunits could be involved in interaction. A structural comparison between the core parts of green plant and cyanobacterial PSII shows differences in the position of the cyt b559 subcomplex and of the CP47 core antenna with respect to the D1/D2 reaction centre. Within the D1/D2 proteins, there are clear differences between plants and cyanobacteria at the stromal ends of membrane-spanning $\alpha$-helices, even though these proteins are highly homologous. The differences in subunit arrangements in these two complexes may reflect an adaptation to the peripheral antenna systems, membraneextrinsic phycobilisomes in the case of cyanobacteria and membrane-bound peripheral LHCII subunits in the case of green plants (Büchel and Kühlbrandt 2005).

State transitions are a physiological adaptation mechanism that changes the interaction of the peripheral antenna proteins with the PSI and PSII core complexes. In green plants and green algae, membrane-bound peripheral antenna proteins play a specific mobile role to (re)direct part of the excitation energy either towards PSI or PSII ; whereas in cyanobacteria the phycobilisomes are considered to play this role. It was found with FRAP measurements that the phycobilisomes diffuse quite rapidly, but that PSII is immobile on the timescale of the measurement, indicating that the linkage between PBSs and the photosystems is unstable (Sarcina et al. 2001, 2006; Joshua et al. 2005). Based on these data it was proposed that the lateral diffusion of PBSs is involved in regulation of photosynthetic light-harvesting (state 1-state 2 transitions) (Mullineaux et al. 1997). It was found that phycobilisomes diffuse, on average, nearly three times faster in a PsaL(-) mutant than in the wildtype (Aspinwall et al. 2004). It is currently believed that light-induced state transition depends completely on a movement of PBSs towards PSI or PSII ( $\mathrm{Li}$ et al. 2006).

Despite the fact that high-resolution data on the interaction of PBSs and photosystems are lacking, models for their organization have been presented (Bald et al. 1996). For hemidiscoidal phycobilisomes the key features are the PSII dimer as prerequisite for interaction and a row-like organization of PSII dimers as a template for phycobilisome attachment. Two PSII monomers form the 'contact area' within the PSII-PBS supercomplex under so-called 'state 1' conditions. In this model attachment of PBS with Photosystem I (PSI) under so-called 'state 2' conditions was proposed (Bald et al. 1996). This model stresses that the multimeric state of the PSI and PS II complexes will be of relevance. In cyanobacteria, PSI can be present as monomers or trimers and PSII as monomers or dimers. In red algae, both PSI and PSII are different because they bind several copies of membrane-associated antenna copies, which are lacking in cyanobacteria (S. Kerë̈che, R. Kouřil, G.T. Oostergetel, A. B. Doust, E.J. Boekema, J.P. Dekker, unpublished data). Moreover, PSI only exists as a monomer (Gardian et al. 2007).

We have studied the supramolecular conformation of hemiellipsoidal PBSs in Porphyridium cruentum, as well as-for comparison-hemidiscoidal PBS in a Synechocystis PCC 6803 mutant by single particle electron microscopy. PBS-thylakoid vesicles provide relatively uniform PBS structure in contrast to isolated PBSs, and allow us to acquire a spatial view of hemiellipsoidal structure. It is suggested that one PBS is likely to associate and line-up with one PSII dimer, in both cyanobacteria and red algea despite the fact that the red algal PBSs are two times larger in width. The larger PBS width in red algae can, however, be compensated by a membrane-bound peripheral antenna, absent in cyanobacteria.

\section{Materials and methods}

Isolation of isolated PBSs and PBS-thylakoid membranes

Porphyridium cruentum was grown in an artificial sea water medium. Flasks were supplied with $3 \% \mathrm{CO}_{2}$ in air through a plug of sterile cotton at a constant temperature of $18^{\circ} \mathrm{C}$. Cultures were illuminated continuously with light provided by daylight fluorescent lamps at different light intensities, $6 \mathrm{Wm}^{-2}$ (low-light) and $15 \mathrm{Wm}^{-2}$ (high-light). The separation of isolated PBSs followed a published protocol (Glazer 1988).

The PBS-membrane vesicles were prepared as described by Mustardy et al. (1992) with slight modifications. Cells were harvested by centrifugation $(5000 \mathrm{~g} ; 10 \mathrm{~min})$, rinsed 
with $0.5 \mathrm{M}$ potassium phosphate buffer ( $\mathrm{pH} 7.0)$, and then suspended in SPC medium (0.5 M sucrose, citrate, $0.5 \mathrm{M}$ potassium phosphate, $0.3 \mathrm{M}$ potassium citrate, $\mathrm{pH}$ 7.0). After homogenization, the cells were broken in a French press at 4,000 psi in order to achieve large membrane sheets with minimal disturbance. The broken cell mixture was layered on a two-step sucrose gradient (1.0 M and $1.3 \mathrm{M})$ with the same buffer conditions as the cell suspension. After centrifugation for $30 \mathrm{~min}$ in a microcentrifuge, the PBS-containing thylakoids were collected from the 1.0-1.3 M sucrose interface.

Samples for imaging were prepared by placing glowdischarged, collodion-carbon-coated copper grids (200 mesh/inch) onto a droplet of sample solution for $10 \mathrm{~min}$, excess liquid was then blotted off and the samples were fixed with $2 \%$ glutaraldehyde, followed by successive washes on three droplets of double-distilled water. Staining was achieved by placing the grid on a droplet of $2 \%$ uranyl acetate. Finally, the staining solution was blotted off.

\section{Electron microscopy}

Electron microscopy was performed on a CM120 electron microscope (Philips, Eindhoven, The Netherlands) operated at $120 \mathrm{kV}$. Images were recorded under low dose conditions (a total dose $\sim 25 \mathrm{e}^{-} / \AA^{2}$ ) with a 4,000 SP $4 \mathrm{~K}$ slow-scan CCD camera (Gatan, Pleasanton, CA). Images of 2-D arrays of membrane-bound phycobilisomes were recorded at 45,000 magnification, at a defocus value of $600 \mathrm{~nm}$ with a pixel size of $6.5 \AA$ at the level of the specimen, (after binning the images). From the sample of non-crystalline phycobilisomes, 600 images were recorded at 80,000 magnification, at a defocus value of $-380 \mathrm{~nm}$ with a pixel size of $3.75 \AA$ at the level of the specimen after a binning of 2 . In total, about 12,000 single particle projections were selected for single particle image analysis, which was performed with the Groningen Image Processing (GRIP) software package on a PC cluster. Selected single-particle projections were aligned by multi-reference and reference-free alignment procedures (Penczek et al. 1992; van Heel et al. 2000). Particles were then subjected to multivariate statistical analysis followed by hierarchical classification (van Heel et al. 2000).

\section{Results}

Membranes with attached PBSs were studied with electron microscopy. By cross-linking with gluteraldehyde, the integrity of the PBS organization on the membrane was preserved. Under low-light growth conditions $\left(6 \mathrm{~W} / \mathrm{m}^{-2}\right)$, the PBSs on the membrane were mostly arranged in ordered domains with a high packing density of about $560 \pm 20$ PBSs per $\mu \mathrm{m}^{2}$ (Fig. 1, left frame). In contrast, at higher light intensities the distribution of PBSs is largely disordered, and the density is significantly lower, $384 \pm 45$ PBSs per $\mu \mathrm{m}^{2}$ (Fig. 1, right frame). This is in agreement with earlier reports of the dependence of pigment density in P. cruentum on growth conditions (Jahn et al. 1984; Gantt et al. 2003), but this is the first time that it is shown that the PBS arrangement is also affected.

Single particle analysis was performed on large sets of electron microscopy projections, selected from EM specimens with negatively stained membranes, on which many PBSs were still membrane-bound and smaller numbers lying around the membranes. The best preserved details were found in the top view of the membrane-bound PBS, which has maximal dimensions of $60 \times 41 \mathrm{~nm}$ in projection (Fig. 2A). Free PBSs have about the same features but lack sharp details (Fig. 2B). A trapeziform projection shows the PBS in a side view position, but it appears rather fuzzy, despite the fact that over 4,000 projections were processed (Fig. 2C). Smaller projections are almost featureless (Fig. 2D, E). A small, almost circular-shaped projection with 3-fold symmetry and a diameter of about $11.5 \mathrm{~nm}$ is a common impurity and is very likely to be a PBS fragment released during storage or EM specimen
Fig. 1 Typical examples of negatively stained

cyanobacterial membranes and associated phycobilisomes showing multiple ordered 2-D arrays under low-light conditions (left frame) and a random distributiom under high-light conditions (right frame) and the space bar equals $1 \mu \mathrm{m}$ for the left frame and $400 \mathrm{~nm}$ for the right frame
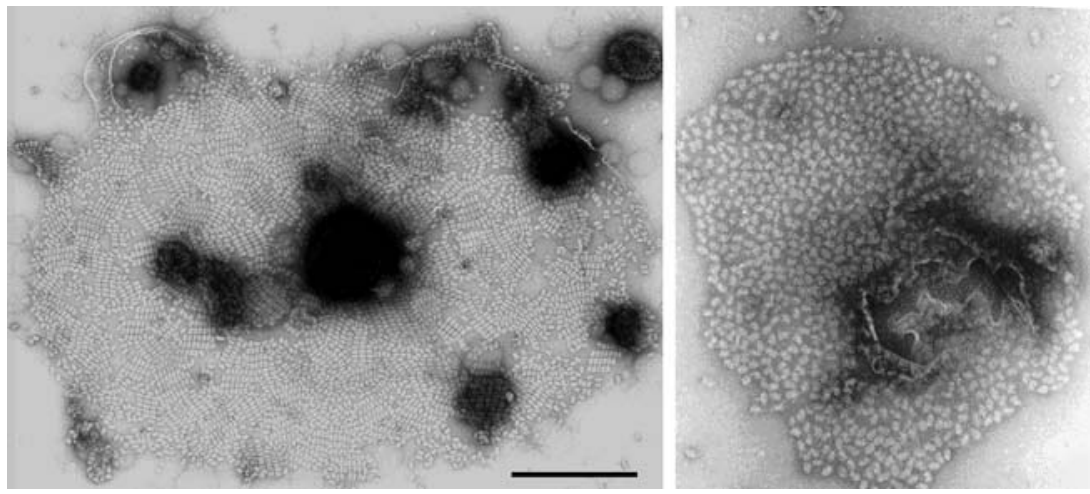


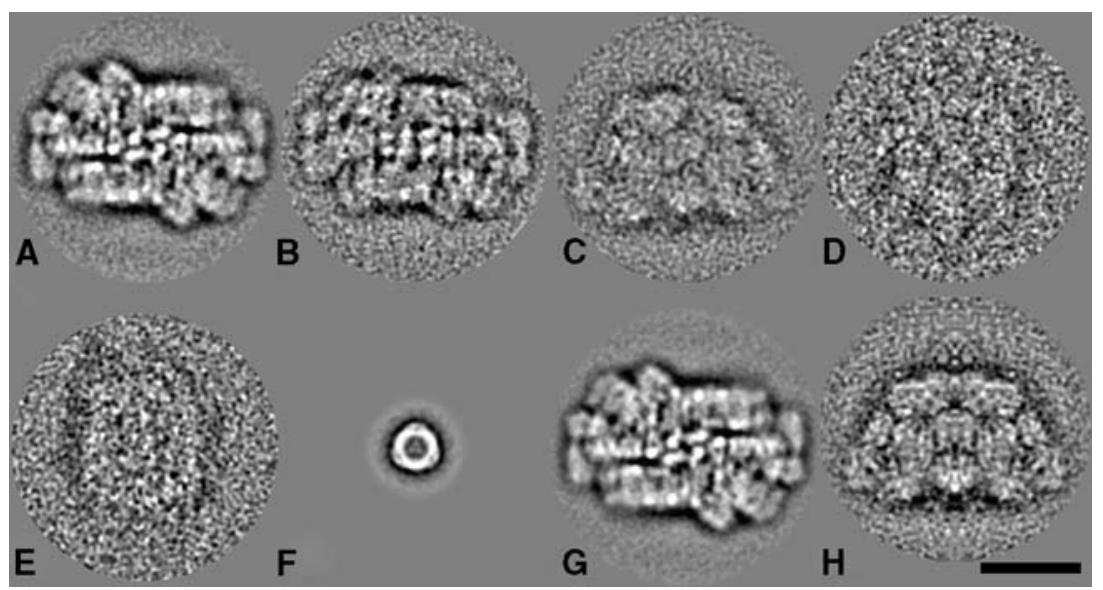

Fig. 2 Single particle electron microscopy of phycobilisome projections from Porphyridium cruentum (A) View in the membrane plane of membrane-bound phycobilisomes (B) View in the membrane plane of free phycobilisomes $(\mathbf{C}-\mathbf{E})$ side views of free phycobilisomes. $(\mathbf{F})$ Most common phycobilisome fragment. (G) image of A with 2-fold

preparation (Fig. 2F). Presumably this is a single disc of phycobiliproteins with a linker polypeptide incorporated as can be discerned from the electron density in the centre.

In an attempt to study the types of packing of PBSs within 2D arrays we compared several hundred areas. In parts with a low amount of ordering the PBSs have a tendency to associate into specific linear structures with their long edges. This is also true for those arrays with the rotational symmetry imposed after analysis (H) Image of $\mathrm{C}$, with mirror symmetry imposed after analysis. The number of summed projections for A-F is 1520, 484, 4096, 380, 499 and 746, respectively. The space bar is $25 \mathrm{~nm}$

highest amount of ordering. These membrane parts do not show a uniform type of packing, as is exemplified by two average sums of arrays showing a different repeating motif and size, which is about $55 \times 35 \mathrm{~nm}$ in Fig. $3 \mathrm{C}$ and $45 \times 31 \mathrm{~nm}$ in Fig. 3D, respectively.

Since there may be specific interactions of the PBSs and the underlying membrane arrangement of PSII, we also compared these arrays with the dimensions of PSII and
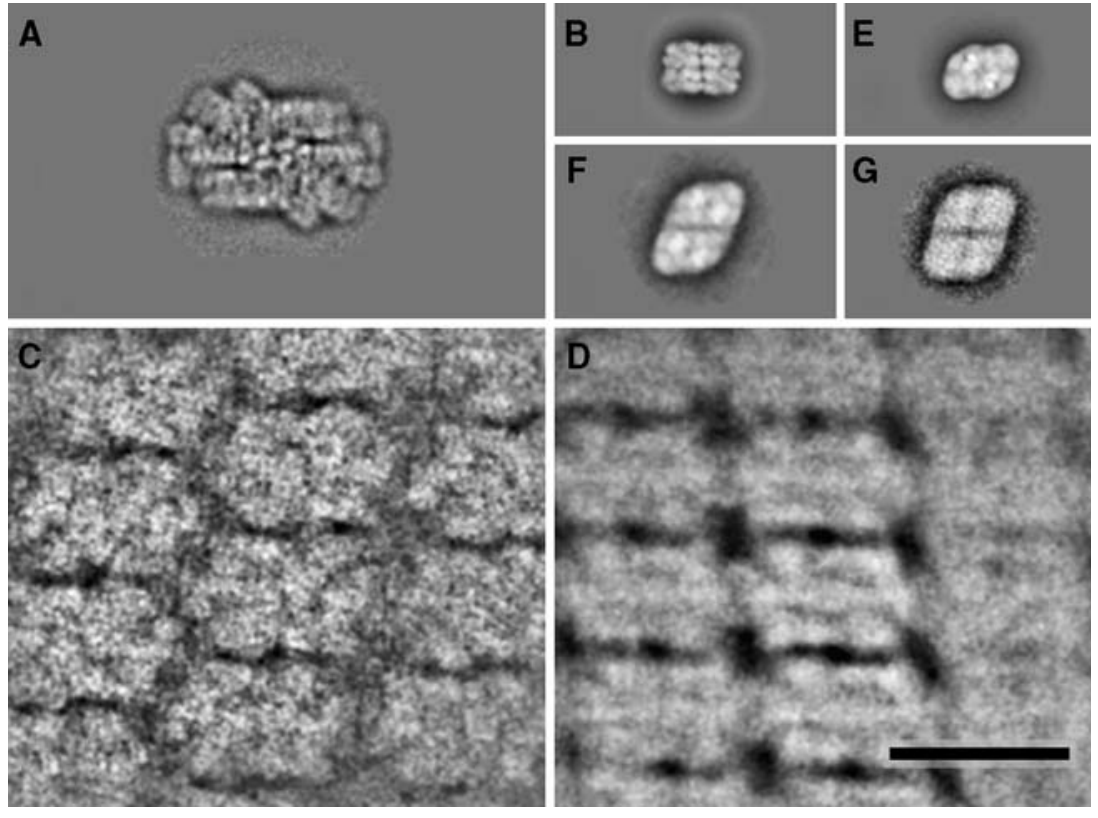

Fig. 3 Comparison of semi-crystalline arrays of phycobilisomes. (A) the membrane-attached phycobilisome (B) APC core structure of Synechocystis PCC 6803 ck mutant, sum of 5019 projections for comparison (A.A. Arteni, R. Ajlani, E.J. Boekema, unpublished results). (C) $2 \mathrm{D}$ array with semi-crystalline phycobilisomes, an average of 11 fragments (D) 2D array with semi-crystalline

phycobilisomes, an average of 107 fragments (E) PSII dimer (from Thermosynechococcus elongatus) (F) PSII double dimer, found after solubilization of membranes with dodecyl maltoside, a sum of 59 projections (G) PSII double dimer, found after solubilization of membranes with digitonin, a sum of 46 projections. The space bar is $50 \mathrm{~nm}$ 
with those of a hemi-discoidal type PBSs of cyanobacteria. The width of cyanobacterial PBSs can be derived accurately from the central PBS component, which is the APC core structure. In the top view position, the APC core structure has a length and width of 22.5 and $15 \mathrm{~nm}$, respectively (Fig. 3B). The width is about the same of a PSII core dimer (Fig. 3E). During previous structural studies on PSII from Thermosynechococcus elongatus we have observed small numbers of a higher type of association of PSII, which were double dimers (Fig. 3F). These dimers are also present in low numbers after membrane solubilization with digitonin, without further purification steps (Fig. 3G; Folea and Boekema, unpublished data).

\section{Discussion}

Single particle electron microscopy revealed the overall features of the hemispherical PBS of Porphyridium cruentum. The PBSs attached to the membranes have the best preserved features, whereas the side views of free PBSs appear fuzzy, indicating some amount of structural flexibility. Some of the flexibility could have been caused by the air drying of the negatively stained EM specimens. On the other hand, the packing of two types of 2D arrays also differs, because the total surface in one type of packing is substantially smaller than in the other one (Fig. 3), suggesting intrinsic flexibility.

The supramolecular organization of the red algal and cyanobacterial membranes is not understood in detail, because we do not know how the PBSs are precisely associated to PSII and PSI. There is for instance no information on the subunits involved in this interaction. Some low-resolution interaction concepts are based on freeze-fracture electron microscopy, which has revealed that cyanobacterial PSII can be arranged in a row-like organization in the membrane with a repeat of 10-12 nm (Mörchel and Schatz 1987) or $14 \mathrm{~nm}$ (Nilsson et al. 1992). The double dimers presented in Fig. 3 and first discussed in Kuhl et al. 1999, are considered to be remnants of the rowlike PSII organization. Based on these data, a model for the arrangement of PBSs was proposed as described in Bald et al. (1996). In this model the PBSs associate along the rows with their short axis as a repeat. This model makes sense for photosynthetic organisms with hemi-discoidal PBSs because there is a 1:1 match between the $15 \mathrm{~nm}$ width of the APC core, as presented in Fig. 2, and the PSII dimmer, which has about the same width.

The situation in the red alga $P$. cruentum is, however, obviously different because of the much larger size of its PBSs. It could have a different or no special arrangement of its PBSs in relation to row-like PSII. In particular it was shown that the tricylindrical core of the PBSs in
P. cruentum is two times wider than that of the hemidiscoidal forms in cyanobacteria (Redecker et al. 1993), which we confirm in the current study. This is commensurate with the suggestion that the PBSs in red algae bind to a tetrameric PSII complex, on the basis of freeze-fracture EM imaging (Redecker et al. 1993; Teckos et al. 2004). Further corroboration is derived from the stoichiometry of PBSs and PSII complexes which is about 1:3-4 (Cunningham et al. 1989). These authors show that the stoichiometry of the photosystems and PBSs in P. cruentum is largely unaffected by light intensity (Cunningham et al. 1989).

However, we have to take into account that red algal PSI and PSII have a peripheral membrane-embedded antenna system that makes the total PSI and PSII supercomplexes substantially larger in surface area. The precise arrangement of the peripheral antanna in red algal PSII is unknown, but it was recently found that in the related unicellular cryptophyte Rhodomonas SC 24 the peripheral antenna extends the width of PSII by $50 \%$ on one of its sides (S. Kereiche, R. Kouřil, G.T. Oostergetel, A. B. Doust, E.J. Boekema, J.P. Dekker, unpublished data, see Appendix I). If such an antenna arrangent would also exist on both sides of $P$. cruentum PSII, the width would have been doubled to about $30 \mathrm{~nm}$, and become similar to the PBS width in one of the 2-D arrays (Fig. 3D). This would mean that a 1:1 ratio between rows of PSII and PBSs is also possible in red algea, despite the giant dimensions of the PBSs. This would be a meaningful ratio for light-harvesting under low-light conditions, where ordered arrays have been observed. But whether a $1: 1$ or a lower 1:2-4 ratio is true or not needs to be established by further work on the supramolecular organization of PSII within the membrane and by analyzing the size and shape of supercomplexes composed of PSII and its peripheral antenna by single particle electron microscopy.

Acknowledgements We thank Dr. G. Ajlani for providing samples of the Synechocystis PCC 6803 ck mutant. We thank Dr. W. Keegstra for his invaluable help in image analysis. TJA and LNL acknowledge expert assistance and advice from Dr. G.E.M. Lamers on electron microscopy. This project is part of the research programme "From Molecule to Cell" funded by the Netherlands Organization for Scientific Research (NWO) and the Foundation for Earth and Life Sciences (ALW).

\section{References}

Adir N (2005) Elucidation of the molecular structures of components of the phycobilisome: reconstructing a giant. Photosynth Res 85:15-32

Aspinwall CL, Sarcina M, Mullineaux CW (2004) Phycobilisome mobility in the cyanobacterium Synechococcus sp PCC7942 is influenced by the trimerisation of Photosystem I. Photosynth Res 79:179-187 
Bald D, Kruip J, Rögner M (1996) Supramolecular architecture of cyanobacterial thylakoid membranes: how is the phycobilisome connected with the photosystems? Photosynth Res 49:103-118

Barber J, Morris EP, da Fonseca PCA (2003) Interaction of the allophycocyanin core complex with photosystem II. Photochem Photobiol Sci 2:536-541

Betz M (1997) One century of protein crystallography: the phycobiliproteins. Biol Chem 378:167-176

Büchel C, Kühlbrandt W (2005) Structural differences in the inner part of Photosystem II between higher plants and cyanobacteria. Photosynth Res 85:3-13

Cunningham FX, Dennenberg RJ, Mustardy L, Jursinic PA, Gantt E (1989) Stoichiometry of Photosystem I, Photosystem II, and phycobilisomes in the red alga Porphyridium cruentum as a function of growth irradiance. Plant Physiol 91:1179-1187

Gantt E (1980) Structure and function of phycobilisomes: light harvesting pigment complexes in red and blue-green algae. Int Rev Cytol 66:45-80

Gantt E (1986) Phycobilisomes. In: Staehelin LA, Anderson JM,Arntzen CJ (eds) Photosynthesis III: Photosenthetic membranes and light harvesting systems. Springer, pp 260-268

Gantt E, Conti SF (1966) Granules associated with the chloroplast lamellae of Porphyridium cruentum. J Cell Biol 29:423-434

Gantt E, Lipschultz CA, Zilinskas B (1976) Further evidence for a phycobilisome model from selective dissociation, fluorescence emission, immunoprecipitation, and electron microscopy. Biochim Biophys Acta 430:375-388

Gantt E, Grabowski B, Cunningham FX Jr (2003) Antenna systems of red algae: phycobilisomes with Photosystem II and chlorophyll complexes with Photosystem I. In: Green BR, Parson WW (eds) Light-harvesting antennas in photosynthesis. Kluwer Academic Publishers, Netherlands, pp 307-322

Gardian Z, Bumba L, Schrofel A, Herbstova M, Nebesarova J, Vacha F (2007) Biochim Biophts Acta 1767:725-731

Glazer AN (1988). Phycobilisomes. In: Packer L, Glazer AN (eds) Methods in enzymology. Academic Press, San Diego, pp 304-312

Grossman AR, Bhaya D, Apt KE, Kehoe DM (1995) Light-harvesting complexes in oxygenic photosynthesis: diversity, control, and evolution. Annu Rev Genet 29:231-288

Grossman AR, Schaefer MR, Chiang GG, Collier JL (1993) The phycobilisome, a light-harvesting complex responsive to environmental conditions. Microbiol Rev 57:725-749

Jahn W, Steinbiss J, Zetsche K (1984) Light intensity adaptation of the phycobiliprotein content of the red alga Porphyridium. Planta 161:536-539

Joshua S, Bailey S, Mann NH, Mullineaux CW (2005) Involvement of phycobilisome diffusion in energy quenching in cyanobacteria. Plant Physiol 138:1577-1585

Kuhl H, Rögner M, van Breemen JFL, Boekema EJ (1999) Localization of cyanobacterial photosystem II donor-side subunits by electron microscopy and the supramolecular organization of photosystem II in the thylakoid membrane. Eur $\mathrm{J}$ Biochem 266:453-459

Li H, Li DH, Yang SZ, Xie H, Zhao JQ (2006) The state transition mechanism-simply depending on light-on and -off in Spirulina platensis. Biochim Biophys Acta 1757:1512-1519

Liu LN, Chen XL, Zhang YZ, Zhou BC (2005) Characterization, structure and function of linker polypeptides in phycobilisomes of cyanobacteria and red algae: an overview. Biochim Biophys Acta 1708:133-142

MacColl R (1998) Cyanobacterial phycobilisomes. J Struct Biol 124:311-334

Mörchel E, Schatz GH (1987) Correlation of photosystem II complexes with exo-plasmatic freeze-fracture particles of thylakoids of the cyanobacterium Synechococcus sp. Planta 172:145-154

Mullineaux CW, Tobin MJ, Jones GR (1997) Mobility of photosynthetic complexes in thylakoid membranes. Nature 390:421-424

Nilsson F, Simpson DJ, Jansson C, Andersson B (1992) Ultrastructural and biochemical characterisation of a Synechocystis 6803 mutant with inactivated psbA genes. Arch Biochem Biophys 295:340-347

Redecker D, Wehrmeyer W, Reuter W (1993) Core substructure of the hemiellipoidal phycobilisomes of the red alga Porhyridium cruentum. Eur J Cell Biol 62:442-450

Samsonoff WA, MacColl R (2001) Biliproteins and phycobilisomes from cyanobacteria and red algae at the extremes of habitat. Arch Microbiol 176:400-405

Sarcina M, Tobin MJ, Mullineaux CW (2001) Diffusion of phycobilisomes on the thylakoid membranes of the cyanobacterium Synechococcus 7942. Effects of phycobilisome size, temperature and membrane lipid composition. J Biol Chem 276:46830-46834

Sarcina M, Bouzovitis N, Mullineaux CW (2006) Mobilization of photosystem II induced by intense red light in the Cyanobacterium Synechococcus sp PCC7942. Plant Cell 18:457-464

Sidler WA (1994). Phycobilisome and phycobiliprotein structures. In: Bryant DA (ed) The molecular biology of cyanobacteria. Kluwer Academic Publishers, Dordrecht, pp 139-216

Penczek P, Radermacher M, Frank J (1992) 3-Dimensional reconstruction of single particles in ice. Ultramicroscopy 40:33-53

Van Heel M, Gowen B, Matadeen R, Orlova EV, Finn R, Pape T, Cohen D, Stark H, Schmidt R, Schatz M, Patwardhan A (2000) Single-particle electron cryo-microscopy: towards atomic resolution. Q Rev Biophys 33:307-369

Yi ZW, Huang H, Kuang TY, Sui SF (2005) Three-dimensional architecture of phycobilisomes from Nostoc flagelliforme revealed by single particle electron microscopy. FEBS Lett 579:3569-3573 